\title{
Lattice Boltzmann Method for Modelling of Biological Phenomena
}

\author{
Romain Noël*, Fei Ge , Yue Zhang $^{\dagger}$, Laurent Navarro* and Guy Courbebaisse ${ }^{\ddagger}$ \\ * École Nationale Suprieure des Mines de Saint-Etienne, CIS-EMSE, \\ INSERM U1059 F4200 SAINBIOSE F-42023, CNRS UMR 6158 LIMOS, Saint Etienne, France \\ Email: romain.noeldemse.fr; navarrodemse.fr \\ $\dagger$ Veterans Affairs Medical Center \\ San Francisco, California 94121, USA \\ Email: y. zhang525@gmail.com \\ $\ddagger$ Univ. Lyon, INSA-Lyon, Université Claude Bernard Lyon 1, UJM Saint-Étienne, CNRS, INSERM, \\ CREATIS UMR 5220, U1206, F69621, Lyon, France \\ Email: fgedcreatis.insa-lyon.fr; guy.courbebaissedcreatis.insa-lyon.fr
}

\begin{abstract}
This paper suggests a new methodology based on the Lattice Boltzmann Method for the modelling of complex biomechanical systems. The LBM can be applied for different operations due to the matching of the pixels of medical images with the nodes of the lattice used by the Lattice Boltzmann method. This allows the optimisation and reduction of the computation time when solving multiphysics complex phenomena. To demonstrate the efficiency of the chosen approach, the modelling of the thrombosis phenomenon within the cavity of a giant cerebral aneurysm has been implemented. The underlying strategy is to implement the Lattice Boltzmann Method for different operations such as extracting the geometry of a considered aneurysm associated to its parent vessel, solving fluid dynamics governing the blood flow and modelling the thrombus growth.
\end{abstract}

\section{INTRODUCTION}

The Lattice Boltzmann Method (LBM) is famous in fluid dynamics [1] [2] [3], it is especially appreciated for its accuracy, its rapidity and its simplicity, indeed it requires no meshing operation and a regular or irregular network is sufficient [4]. Moreover, LBM shown also its efficiency in image processing [5]. Since an image is a regular grid of pixels, it is theoretically possible to work on the same network for this two previous fields. Here is presented a method going from the theory to the demonstration of its feasibility through a medical application and even further by adding biological phenomena. Thus, the original idea of working on a same support to implement concurrently image processing, fluid mechanics simulation and biomechanics modelling is original, challenging and promising (see Fig. 1). Thanks to the linearisation of some partial differential equation introduced by Bhatnagar, Gross and Krook [6], it is possible to have a generic LBM capable of solving image processing, computational fluid dynamics and mechanobiology problems. The proposed method is more than a simple succession of well studied cases, it shares the same support to solve all theses problems together. This allows, each LBM layer of the solver to have, at any time, access to informations from the others layers. The presentation of this paper is constructed with the following parts. First a short but general introduction to the LBM method is given.

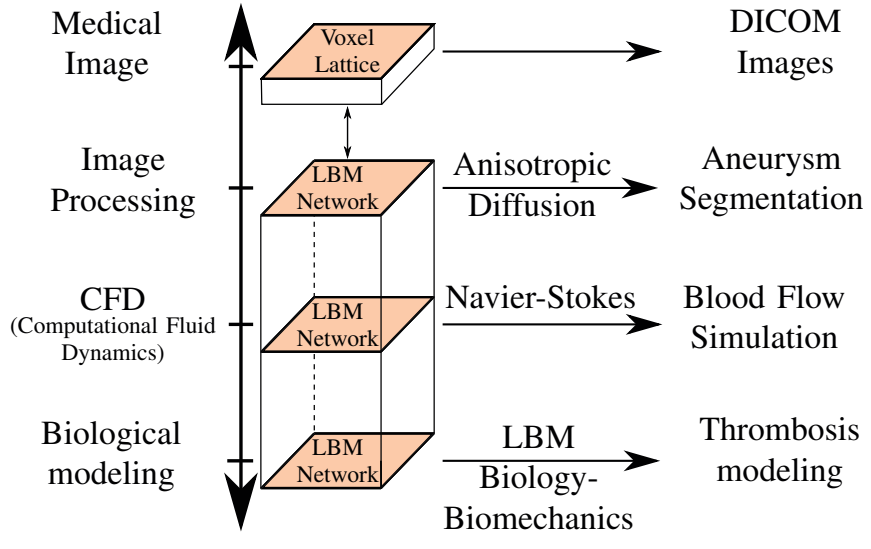

Fig. 1. Schematic representing the order to use the different LBM layers

Second, the LBM is presented as a dedicated tool for image processing, filtering and segmenting images. The third part shows how the LBM can be used as Computational Fluid Dynamics (CFD) applications solver. Finally, the last part shows how LBM can be used to simulate mechanobiogical interactions. Then the application case of a cerebral aneurysm is treated to illustrate the generic proposed method. A cerebral aneurysm is a vascular disorder characterised by a dilation of the vessel wall itself caused by vessel walls weakening. A cerebral aneurysm healing is conditioned by the formation of clot or thrombus. The modelling of this biomechanical phenomenon named thrombosis is complex since the hemodynamic must be coupled to a biomechanical model in order to understand the thrombus formation based on patient biological factors [7] [8].

\section{LATTICE BOLTZMANN METHOD}

\section{A. The Boltzmann equation and the BGK approximation}

In physics, it is common to focus on pressure and temperature parameters. Both are originated from a statistical average over a large number of individual particles. This statistical 
aspect has been taken into account by the Boltzmann equation, which has been proved to be a valuable tool that is best suited to fluids behaviours investigations [9]. The Boltzmann equation takes in consideration the probability at time $t$ that a high level of particles fills a small domain centred in $\boldsymbol{x}$ with a velocity $\boldsymbol{\xi}$. This probability considered as a distribution function $f(\boldsymbol{x}, \boldsymbol{\xi}, t)$ obeys the Boltzmann integro-differential equation [9]:

$$
\frac{\partial f}{\partial t}+\boldsymbol{\xi} \cdot \nabla_{x} f+\frac{\boldsymbol{g}}{m} \cdot \nabla_{\boldsymbol{\xi}} f=\Omega(f, f)
$$

where $\boldsymbol{g}$ is the external force acting on the particles in the fluid, $m$ the particle mass and $\Omega$ the collision integral which describes the collisions between particles. The main concern when working with the Boltzmann equation is the non linear expression of $\Omega$. To tackle this issue, different models have been implemented; In particular the linearisation induced by the BGK model has been proposed by Bhatnagar, Gross and Krook [6]. With this model the right hand side of Eq. (1) becomes:

$$
\frac{\partial f}{\partial t}+\boldsymbol{\xi} \cdot \nabla_{\boldsymbol{x}} f+\frac{\boldsymbol{g}}{m} \cdot \nabla_{\boldsymbol{\xi}} f=-\frac{1}{\tau}\left(f-f^{e q}\right),
$$

where $\tau$ is a relaxation time. In Eq. (2) the MaxwellBoltzmann equilibrium distribution function $f^{e q}$ is expressed as

$$
f^{e q}=\frac{\rho}{\left(2 \pi k_{B} T\right)^{D / 2}} \exp \left[-\frac{(\boldsymbol{\xi}-\boldsymbol{u})^{2}}{2 k_{B} T}\right],
$$

where $\rho$ is the fluid density, $k_{B}$ the Boltzmann constant and $D$ the physical dimension. $\boldsymbol{u}$ is the mean velocity. The temperature $T$ is supposed constant in our problem,

\section{B. From Boltzmann equation to Lattice Boltzmann method}

The LBM is issued from lattice gas cellular automata [9] [10] [11] introduced with the HPP model [12] [13]. The traditional CFD methods aim to solve partial differential equations on a meshed geometry, where the LBM fills the physical space with a regular lattice and models fluid on it by using probable distributions of particles to perform propagation and collision processes over this lattice. Moreover, each node of the lattice respects conservation laws which guarantee to solve correctly fluid mechanics applications. LBM can also be considered as a discretised version of the Boltzmann equation [14] elaborated as follows; Initially, the discretisation of velocity and distribution functions is performed, leading to:

$$
\boldsymbol{\xi} \rightarrow \boldsymbol{\xi}_{i}, \quad(\boldsymbol{x}, t) \rightarrow f_{i}\left(\boldsymbol{x}_{i}, t\right) .
$$

In this operation, the discrete velocities must be correctly chosen to respect the spatial isotropy of the problem [2] [9]. Then, assuming no external force and the BGK approximation, the discrete Boltzmann equation is expressed like:

$$
\frac{\partial f_{i}}{\partial t}+\boldsymbol{\xi}_{i} \cdot \nabla f_{i}=-\frac{1}{\tau}\left(f_{i}-f_{i}^{e q}\right),
$$

Note that $e_{i}=\frac{\xi}{\mathrm{U}_{0}}$ where $\mathrm{U}_{0}$ is the reference velocity. The discrete time unit can be given as $\Delta t=\frac{\Delta x}{c_{s}}=1$ with $\Delta x$ the basic unit for lattice spacing and $c_{s}$ is the speed of sound. $f_{i}^{e q}$ represents the discrete form of the equilibrium distribution function.

After calculus of the Boltzmann equation in nondimensional form, and discretisation of both time and space, the lattice Boltzmann equation expression is obtained [9]:

$$
f_{i}\left(\boldsymbol{x}+\boldsymbol{e}_{i} \cdot \Delta t, t+\Delta t\right)-f_{i}(\boldsymbol{x}, t)=-\frac{1}{\tau}\left(f_{i}-f_{i}^{e q}\right),
$$

where $f_{i}^{e q}$ is obtained with the first terms of Taylor's series of the Maxwellian equilibrium distribution function shown in Eq. (3). It can be read as

$$
f_{i}^{e q}=\rho w_{i}\left(1+\frac{\boldsymbol{e}_{i} \cdot \boldsymbol{u}}{c_{s}^{2}}+\frac{\left(\boldsymbol{e}_{i} \cdot \boldsymbol{u}\right)^{2}}{2 c_{s}{ }^{2}}-\frac{\boldsymbol{u} \cdot \boldsymbol{u}}{2 c_{s}^{2}}\right),
$$

where $w_{i}$ are the weight coefficients associated to the directions; $\rho$ represent the macroscopic density of the fluid and $u$ represents its velocity. The density and the velocity are written in function of the $f_{i}$ :

$$
\begin{gathered}
\rho=\sum_{i} f_{i}=\sum_{i} f_{i}^{e q}, \\
\rho \boldsymbol{u}=\sum_{i} \boldsymbol{e}_{i} f_{i}=\sum_{i} \boldsymbol{e}_{i} f_{i}^{e q} .
\end{gathered}
$$

The numerical code has been implemented in the framework of the Palabos project (http://www.palabos.org) which is an opensource CFD solver based on the Lattice Boltzmann Method. The numerical simulations have been performed by using the facilities of the IN2P3 computing centre (National Institute of Nuclear and Particle Physics) from the CNRS (French National Scientific Research Council).

\section{LBM AND SEGMENTATION}

LBM anisotropic diffusion for image processing has been implemented for image preprocessing such as smoothing and filtering operations [5] [15] and segmentation [16] [17]. To explain the genesis of this method, the Chapman-Enskog and Taylor expansions are applied on the LBM Eq. (6) and the Equation of Diffusion is recovered:

$$
\frac{\partial \rho}{\partial t}=\frac{\Delta x^{2}}{3 \Delta t}(\tau-0.5) \nabla^{2} \rho
$$

Then with a variable relaxation time $\tau$, the anisotropic diffusion equation can be reconstructed.

More recently a LBM segmentation tool has been elaborated to capture a Geometric Active contour model. This new method has been applied with success to the segmentation of aneurysms, and relevant scientific results have been published [18]. Thanks to the advances in medical image techniques and reconstruction tools, patient-specific geometries of aneurysms with parent blood vessels, are provided with the LBM (see Fig. 2). 


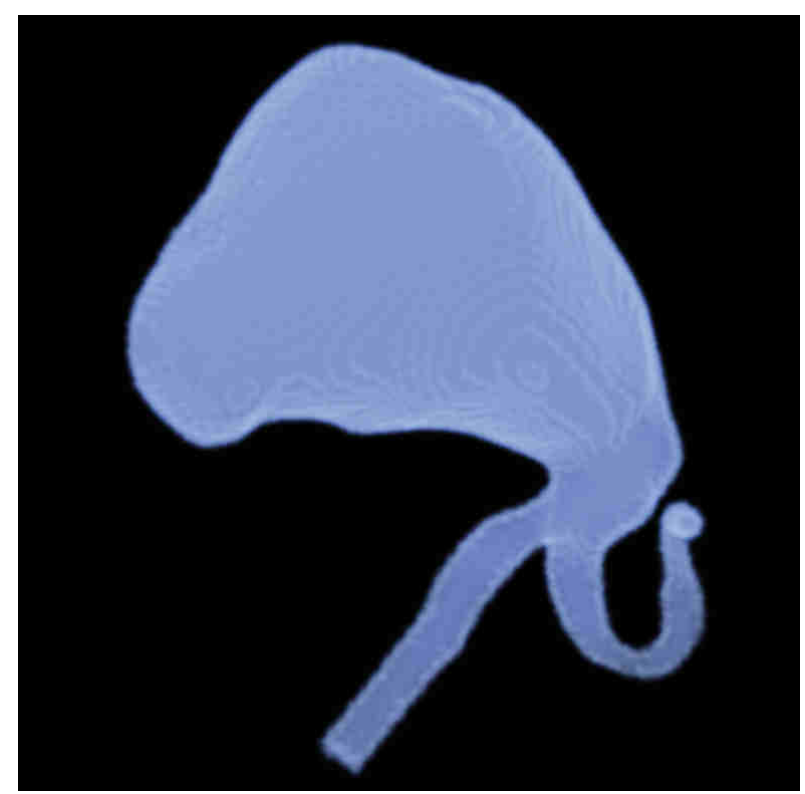

Fig. 2. Result of the thrombus segmentation with a LBM anisotropic diffusion

\section{COMPUTATIONAL FLUID DYNAMICS}

CFD is a branch of fluid dynamics providing a qualitative and quantitative prediction of real fluids by performing numerical solutions of the governing equations. Theses are the famous Navier-Stokes equations which aim to describe the evolution of flow behaviour. In fact, these governing equations are complex with no analytical solution yet in the general case. This is the reason why a numerical approach is often chosen. The LBM offers an original way to solve NavierStokes equations at a mesoscopic scale based on the average of microscopic parameters, through the resulting variables such as $\rho$ and $\boldsymbol{u}$ [3], but also with two major macroscopic quantities the stress tensor $\Pi$ and the equilibrium tensor $\Pi^{e q}$ :

$$
\begin{aligned}
& \Pi_{\alpha \beta}=\sum_{i} e_{i \alpha} e_{i \beta} f_{i} \\
& \Pi_{\alpha \beta}^{e q}=\sum_{i} e_{i \alpha} e_{i \beta} f^{e q}=p \delta_{\alpha \beta}+\rho u_{\alpha} u_{\beta}
\end{aligned}
$$

where $e_{i \alpha}$ is the $\alpha^{\text {th }}$ component of the $e_{i}$ (i.e. $e_{i \alpha}=e_{i} \cdot r_{\alpha}$ with $\boldsymbol{r}_{\boldsymbol{\alpha}}$ a vector of the space base) ; $p$ is the fluid pressure and $\delta_{\alpha \beta}$ is the Kronecker symbol.

By applying the Chapman-Enskog expansion in Eq. (5), the Navier-Stokes equations can be retrieved:

$$
\begin{array}{r}
\partial_{t} \rho+\nabla \cdot(\rho \boldsymbol{u})=0, \\
\partial_{t}(\rho \boldsymbol{u})+\nabla \cdot \boldsymbol{\Pi}=0,
\end{array}
$$

where $\Pi$ is the stress tensor:

$$
\boldsymbol{\Pi}=(p \boldsymbol{I}+\rho \boldsymbol{u} \boldsymbol{u}-2 \nu \boldsymbol{\sigma})
$$

with $\sigma$ the viscous stress tensor and $\nu$ the kinematic viscosity.

The hemodynamics field has been studied using CFD with LBM formulation and several studies demonstrated the LBM

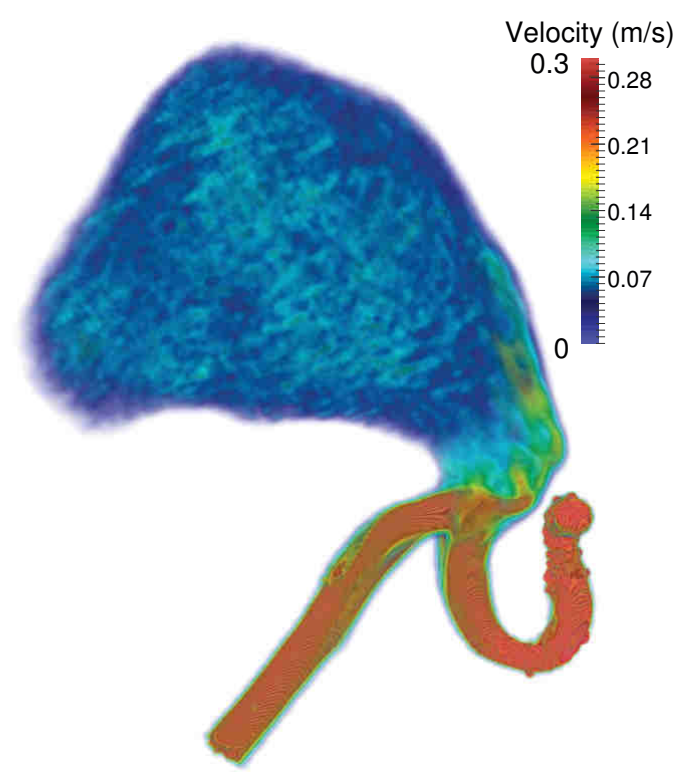

Fig. 3. Computed velocity of the blood flow inside the aneurysm and its parent vessel

ability for providing a detailed analysis of the blood flow for patient-specific cases [8] [19] [20] (see Fig. 3).

\section{THROMBUS MODELLING WITHIN CEREBRAL ANEURYSM}

The biological mechanism of thrombus formation inside aneurysms is complex and deals with different nature of biological parameters with their variability related to patients [21] (see Eq. (16)).

In addition to biochemical factors, hemodynamics plays a key role in the understanding of the thrombus formation [19] [22]. Hemodynamic parameters, such as velocity and Wall Shear Stress (WSS) have been estimated in-vivo and in-vitro to understand the underlying biomechanical reactions [23] [24].

$$
\left\{\begin{array}{l}
\text { Endothelial Cells } \stackrel{\text { WSS }<\text { Threshold }}{\longrightarrow} \text { Thrombin } \\
\text { Thrombin }+ \text { Antithrombin } \rightarrow \emptyset \\
\text { Fibrinogen } \stackrel{\text { Thrombin }}{\longrightarrow} \text { Fibrin } \\
\text { Fibrin } \rightarrow \text { Fibrin Mesh } \\
\text { Fibrin Mesh }+ \text { Platelet } \rightarrow \text { Thrombosis } \\
\text { Thrombosis } \stackrel{\text { Specific time T }}{\longrightarrow} \text { Endothelial Cells }
\end{array}\right.
$$

Thrombus with onion-skin structure have been observed on some giant aneurysms from patients. They have the capacity to initiate the thrombus formation when the WSS inside the cavity of the aneurysm is low enough to provide the good environment for the building of a blood clot [25]. As a result, the thrombus formation can be triggered or not, by changing the magnitude of the velocity profile [20].

In consequence, a thrombosis model has been implemented with the LBM taking in consideration the successive steps 
given by Eq. (16) reflecting the biological schematic on Fig. 4. Indeed, the hemodynamic and the biological parameters are varying [19] [20] [26] [27] [28] with the successive growth of the thrombus (see Fig. 5).

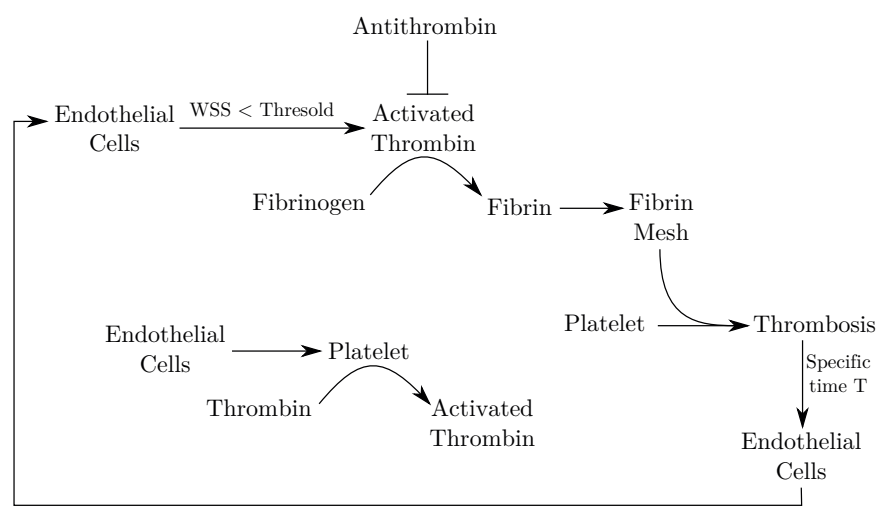

Fig. 4. Schematic of the thrombosis reaction system

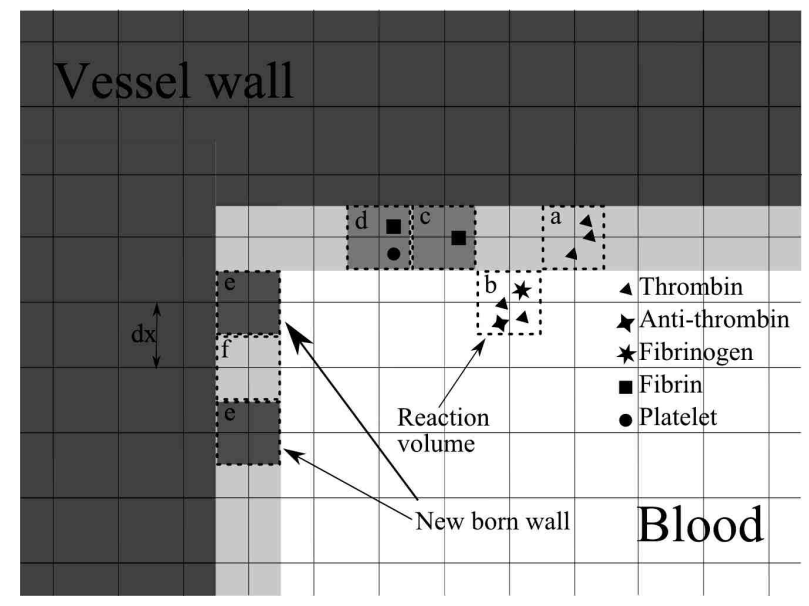

Fig. 5. Numerical simulation of the thrombosis growing layer by layer

In the above model, for example the thrombin particles cannot be generated by a thrombus node until this node becomes a new born wall node after a specific time to control the thrombosis process [26]. Since the considered thrombosis is growing with an onion-skin structure [20], when no thrombin particle stays inside the vessel, the process of constructing the next layer is initiated. This is far from being usual and very different from well studied cases [7] [21] [26].

Thus, a patient-specific giant aneurysm geometry has been chosen for elaborating an onion-skin multilayer model implemented with the LBM [18]. A final result is shown Fig. 6, where the successive layers of the onion-skin clot are delimited by white lines. Comparing with the thrombus developed by the patient, the relevance of the model leads to a good prediction of the formation of the thrombus. This tends to prove the validity of the method and the models used [20].

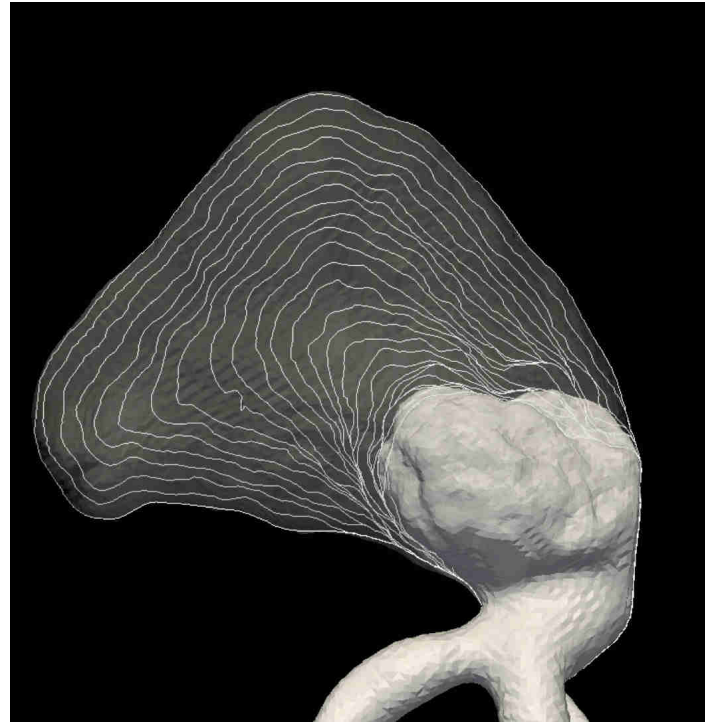

Fig. 6. Representation of the onion-skin structure of the giant aneurysm

\section{Vi. Conclusion}

The Lattice Boltzmann method is powerful by the fact that the physics commonly described through partial differential equations, must be reinterpreted at a mesoscopic scale providing a bridge between microscopic parameters and macroscopic parameters. The fact of solving image processing, CFD and mechanobiology on the same support is original, but above all it has the strong advantage to allow crossing informations at any time. This paper provides the opportunity to imagine the modelling of physico-chemical and biological mechanisms by implementing the Lattice Boltzmann method on a single lattice solving different particulars mechanisms. The efficiency of this idea was illustrated with the thrombus formation, but this method is given without any specific focusing. The challenge for simulating the thrombus formation remains in resolving the biomechanical interactions between different components of the blood taking in consideration the characteristic of the blood flow in the parent blood vessel and in the cavity of the cerebral aneurysm. The presented method gave good results validated by the theoretical frame of CFD and image processing with LBM. Plus, the mechanobiology results were validated by the medical staff of the Thrombus project. The implementation of LBM is a real opportunity to propose an unprecedented and efficient numerical simulation of an onion-skin structured thrombus formation by using the same network/lattice from image processing applied on DICOM medical images, to the modelling of the thrombosis mechanism coupled to the hemodynamics of the blood; leading to a novel investigation tool for medical scenarii without a priori models, based on experimental data.

\section{ACKNOWLEDGEMENT}

This paper has been funded by the European Commission via the Thrombus project FP7-ICT-2009-6-269966 
(http://www.thrombus-vph.eu) and the French National Research Agency via the LBSMI project ANR-15-CE19-0002.

\section{REFERENCES}

[1] S. Chen and G. D. Doolen, "Lattice boltzmann method for fluid flows," Annual review of fluid mechanics, vol. 30, no. 1, pp. 329-364, 1998.

[2] S. Succi, The lattice Boltzmann equation for fluid dynamics and beyond, ser. Numerical mathematics and scientific computation. Oxford : New York: Clarendon Press ; Oxford University Press, 2001.

[3] O. Malaspinas, G. Courbebaisse, and M. Deville, "Simulation of generalized newtonian fluids with the lattice boltzmann method," International Journal of Modern Physics C, vol. 18, no. 12, pp. 1939-1949, 2007.

[4] F. Nannelli and S. Succi, "The lattice Boltzmann equation on irregular lattices," Journal of Statistical Physics, vol. 68 no. 3-4, pp. 401-407, Aug. 1992. [Online]. Available: http://link.springer.com/10.1007/BF01341755

[5] Y. Chen, Z. Yan, and Y. Qian, "An Anisotropic Diffusion Model for Medical Image Smoothing by Using the Lattice Boltzmann Method," in 7th Asian-Pacific Conference on Medical and Biological Engineering, Y. Peng and X. Weng, Eds. Berlin, Heidelberg: Springer Berlin Heidelberg, 2008, vol. 19, pp. 255-259, dOI: 10.1007/978-3-54079039-6_65. [Online]. Available: http://link.springer.com/10.1007/978$3-540-79039-6 \quad 65$

[6] P. L. Bhatnagar, E. P. Gross, and M. Krook, "A Model for Collision Processes in Gases. I. Small Amplitude Processes in Charged and Neutral One-Component Systems," Physical Review, vol. 94, no. 3, pp. 511-525, May 1954. [Online]. Available: http://link.aps.org/doi/10.1103/PhysRev.94.511

[7] A. Tosenberger, F. Ataullakhanov, N. Bessonov, M. Panteleev, A. Tokarev, and V. Volpert, "Modelling of thrombus growth in flow with a DPD-PDE method," Journal of Theoretical Biology, vol. 337, pp. 30-41, Nov. 2013. [Online]. Available: http://linkinghub.elsevier.com/retrieve/pii/S0022519313003469

[8] D. M. Sforza, C. M. Putman, and J. R. Cebral, "Hemodynamics of cerebral aneurysms," Annual Review of Fluid Mechanics, vol. 41, pp. 41-91, 2009.

[9] D. A. Wolf-Gladrow, Lattice-gas cellular automata and lattice Boltzmann models: An Introduction. Springer Science \& Business Media, 2000, no. 1725.

[10] X. He and L.-S. Luo, "Theory of the lattice boltzmann method: From the boltzmann equation to the lattice boltzmann equation," Phys. Rev. E, vol. 56, pp. 6811-6817, Dec 1997. [Online]. Available: http://link.aps.org/doi/10.1103/PhysRevE.56.6811

[11] Y. Qian, D. d'Humières, and P. Lallemand, "Lattice bgk models for navier-stokes equation," EPL (Europhysics Letters), vol. 17, no. 6, p. 479, 1992.

[12] J. Hardy, Y. Pomeau, and O. de Pazzis, "Time evolution of a twodimensional model system. I. Invariant states and time correlation functions," Journal of Mathematical Physics, vol. 14, no. 12, pp. 1746-1759, Dec. 1973. [Online]. Available: http://aip.scitation.org/doi/10.1063/1.1666248

[13] U. Frisch, B. Hasslacher, and Y. Pomeau, "Lattice-Gas Automata for the Navier-Stokes Equation," Physical Review Letters, vol. 56, no. 14, pp. 1505-1508, 1986. [Online]. Available: http://link.aps.org/doi/10.1103/PhysRevLett.56.1505

[14] M. Junk, "A finite difference interpretation of the lattice Boltzmann method," Numerical Methods for Partial Differential Equations, vol. 17, no. 4, pp. 383-402, 2001.

[15] L. Navarro and G. Courbebaisse, "La méthode Lattice Boltzmann en traitement d'image." TAIMA, Hammamet, Tunisie, may 2015. [Online]. Available: http://www.arts-pi.org.tn/taima2015/

[16] Y. Wang, G. Courbebaisse, and Y. M. Zhu, "Segmentation of giant cerebral aneurysms using a multilevel object detection scheme based on lattice boltzmann method," in Signal Processing, Communications and Computing (ICSPCC), 2011 IEEE International Conference on, 2011, pp. 1-4.

[17] Y. Wang, Y. Zhang, L. Navarro, O. F. Eker, R. A. Corredor Jerez, Y. Chen, Y. Zhu, and G. Courbebaisse, "Multilevel segmentation of intracranial aneurysms in ct angiography images," Medical physics, vol. 43, no. 4, pp. 1777-1786, 2016.
[18] Y. Chen, L. Navarro, Y. Wang, and G. Courbebaisse, "Segmentation of the thrombus of giant intracranial aneurysms from CT angiography scans with lattice Boltzmann method," Medical Image Analysis, vol. 18, no. 1, pp. 1-8, Jan. 2014. [Online]. Available: http://linkinghub.elsevier.com/retrieve/pii/S1361841513001175

[19] J. R. Cebral, M. A. Castro, J. E. Burgess, R. S. Pergolizzi, M. J Sheridan, and C. M. Putman, "Characterization of cerebral aneurysms for assessing risk of rupture by using patient-specific computational hemodynamics models," American Journal of Neuroradiology, vol. 26, no. 10 , pp. 2550-2559, 2005.

[20] Y. Zhang, "Hemodynamic investigation and thrombosis modeling of intracranial aneurysms," Ph.D. dissertation, Lyon, INSA, 2015.

[21] R. Ouared, B. Chopard, B. Stahl, D. Rfenacht, H. Yilmaz, and G. Courbebaisse, "Thrombosis modeling in intracranial aneurysms: a lattice Boltzmann numerical algorithm," Computer Physics Communications, vol. 179, no. 1-3, pp. 128-131, Jul. 2008. [Online]. Available: http://linkinghub.elsevier.com/retrieve/pii/S0010465508000283

[22] H.-J. Steiger, "Pathophysiology of development and rupture of cerebral aneurysms." Acta Neurochirurgica Supplementum, vol. 48, pp. 1-57, 1989.

[23] H. J. Steiger, R. Aaslid, S. Keller, and H.-J. Reulen, "Strength, elasticity and viscoelastic properties of cerebral aneurysms," Heart and vessels, vol. 5, no. 1, pp. 41-46, 1989.

[24] M. Toth, G. L. Nadasy, I. Nyary, T. Kerenyi, M. s. Orosz, G. Molnarka, and E. Monos, "Sterically inhomogenous viscoelastic behavior of human saccular cerebral aneurysms." Journal of vascular research, vol. 35, no. 5, pp. 345-355, 1997.

[25] A. Sagar and J. Varner, "Dynamic Modeling of the Human Coagulation Cascade Using Reduced Order Effective Kinetic Models," Processes, vol. 3, no. 1, pp. 178-203, Mar. 2015. [Online]. Available: http://www.mdpi.com/2227-9717/3/1/178/

[26] O. Malaspinas, A. Turjman, D. Ribeiro de Sousa, G. Garcia-Cardena, M. Raes, P.-T. T. Nguyen, Y. Zhang, G. Courbebaisse, C. Lelubre, K. Zouaoui Boudjeltia, and B. Chopard, "A spatio-temporal model for spontaneous thrombus formation in cerebral aneurysms," Journal of Theoretical Biology, vol. 394, pp. 68-76, Apr. 2016. [Online]. Available: http://linkinghub.elsevier.com/retrieve/pii/S002251931600028X

[27] D. R. de Sousa, C. Vallecilla, K. Chodzynski, R. C. Jerez, O. Malaspinas, O. F. Eker, R. Ouared, L. Vanhamme, A. Legrand, B. Chopard, G. Courbebaisse, and K. Zouaoui Boudjeltia, "Determination of a shear rate threshold for thrombus formation in intracranial aneurysms," Journal of neurointerventional surgery, vol. 8, pp. 853-858, 2016.

[28] B. Chopard, D. R. de Sousa, J. Latt, F. Dubois, C. Yourassowsky, P. Van Antwerpen, O. Eker, L. Vanhamme, D. Perez-Morga, G. Courbebaisse et al., "A physical description of the adhesion and aggregation of platelets," Royal Society Open Science, no. 4(4), 170219, 2017. 